\title{
Visual imagery can impede reasoning
}

\author{
MARKUS KNAUFF \\ Freiburg University, Freiburg, Germany \\ and \\ P. N. JOHNSON-LAIRD \\ Princeton University, Princeton, New Jersey
}

\begin{abstract}
Although it is natural to suppose that visual mental imagery is important in human deductive reasoning, the evidence is equivocal. This article argues that reasoning studies have not distinguished between ease of visualization and ease of constructing spatial models. Rating studies show that these factors can be separated. Their results yielded four sorts of relations: (1) visuospatial relations that are easy to envisage visually and spatially, (2) visual relations that are easy to envisage visually but hard to envisage spatially, (3) spatial relations that are hard to envisage visually but easy to envisage spatially, and (4) control relations that are hard to envisage both visually and spatially. Three experiments showed that visual relations slow down the process of reasoning in comparison with control relations, whereas visuospatial and spatial relations yield inferences comparable with those of control relations. We conclude that irrelevant visual detail can be a nuisance in reasoning and can impede the process.
\end{abstract}

"I am by the sea and I have a picture. This is a picture of a picture. I am-" She screwed up her face and scowled"thinking." . . . She paused, frustrated by the vivid detail of her picture, not knowing how to extract from it the significance she felt was there.

- The Inheritors, William Golding, 1955, p. 62

Visual mental images are an important part of human cognition, and it is natural to suppose that they can help us to reason. Various sorts of evidence are compatible with this assumption, including the well-known studies of the mental rotation and the mental scanning of images (Kosslyn, 1980; Shepard \& Cooper, 1982). The aim of the present article, however, is to reexamine this assumption, to reject it, and to propose an alternative hypothesis in its stead. The article is motivated by the distinction between visual and spatial representations and processes that has been made by several researchers (e.g., Johnson-Laird, 1998; Kosslyn, 1994; Landau \& Jackendoff, 1993; Rueckl, Cave, \& Kosslyn, 1989; Ungerleider \& Mishkin, 1982)

\footnotetext{
The present experiments were carried out in part while the first author was a visiting fellow at the Department of Psychology, Princeton University. The research was supported in part by Grants Kn465/2-1 and Kn465/3-2 from the German National Research Foundation(DFG) to the first author and by Grant 0076287 from the National Science Foundation to the second author. The authors are grateful to Elin Arbin, Sam Glucksberg, Uri Hasson, Juan Garcia Madruga, Thomas Mulack, Vladimir Sloutsky, Gerhard Strube, Clare Walsh, Yingrui Yang, and Lauren Ziskind for helpful discussions of the research and to the editor of the journal, Colin MacCleod, and two anonymous reviewers for many useful comments on the manuscript. Correspondence concerning this article should be addressed to M. Knauff, Center for Cognitive Science, Freiburg University, Friedrichstr. 50, 79098 Freiburg, Germany (e-mail: knauff@cognition.iig.uni-freiburg.de).
}

and is supported by investigations with brain-damaged patients (e.g., Newcombe, Ratcliff, \& Damasio, 1987), functional brain imaging studies (e.g., Smith et al., 1995), and experiments on visual and spatial working memory (see the overview in Logie, 1995). In addition, the article focuses on deductive reasoning, in which the truth of the premises ensures the truth of the conclusion, in contrast to inductive reasoning, in which the truth of the premises does not warrant the truth of the conclusion.

We begin with a review of previous studies of imagery and deduction and show that they have often overlooked a possible confounding between materials that invoke visual imagery and materials that invoke spatial representations. We then outline our alternative hypothesis that visual images are not critical for deductive reasoning and may even interfere with the process, whereas spatial representations help individuals to reason deductively. We report three experiments that test this hypothesis. Finally, we draw some general conclusions about visual imagery, spatial representations, and reasoning.

A pioneering study of imagery and deductive reasoning was carried out by De Soto, London, and Handel (1965), who investigated so-called three-term series problems, such as

Ann is taller than Beth.

Cath is shorter than Beth.

Who is tallest?

These authors argued that reasoners represent the three individuals in a visual image and then "read off" the answer by inspecting the image. Huttenlocher (1968) also argued that reasoners carrying out such three-term series problems imagine an analogous physical arrangement of objects. Shaver, Pierson, and Lang (1975) re- 
ported that performance on three-term series problems depends on the ease of imaging the given materials, the instruction to form images, and the participants' ability to form images. Likewise, Clement and Falmagne (1986) found that materials rated as easy to imagine led to fewer errors in verbal reasoning (at least, if the materials related to knowledge). With a different procedure, Pearson, Logie, and Gilhooly (1999) found that a visual secondary task interfered with a primary task of reasoning-related mental synthesis.

In contrast, several studies have failed to detect any effect of imageability on reasoning. Sternberg (1980) found no difference in the accuracy of solving problems that were easy or hard to visualize. Richardson (1987) reported that reasoning with visually concrete problems was no better than reasoning with abstract problems, and similar results are reported in Johnson-Laird, Byrne, and Tabossi (1989) and in Newstead, Pollard, and Griggs (1986). Moreover, Sternberg did not find any reliable correlation between scores on the imageability items of IQ tests and reasoning ability.

One possible resolution of the inconsistency in the previous results from studies of reasoning is that the distinction between visual images and spatial representations has been overlooked. This distinction seems first to have been made by Ungerleider and Mishkin (1982) in their lesion studies with monkeys and by researchers investigating human brain injuries (for an overview, see Newcombe \& Ratcliff, 1989). Both sorts of studies showed that visual and spatial processes are associated with different cortical areas. Additional evidence is provided by experiments in which the resource limitations of working memory are examined (e.g., Logie, 1995) and, most recently, studies using functional brain imaging (D'Esposito et al., 1998; Smith et al., 1995).

What does the distinction between visual and spatial processes mean for reasoning? Johnson-Laird (1998) emphasized that spatially organized mental models are not to be identified with visual images. On the one hand, a relation such as The hat is above the cup is easy to visualize, given a modicum of competence in forming images. But it may also be readily represented spatiallythat is, individuals may construct a spatial model of the relation without any conscious awareness of a visual image. Such a model, according to the theory of mental models, suffices for reasoning: It captures the relevant logical properties. Hence, the transitivity of a relation of the form $A$ is above $B$ derives merely from the meaning of the relation and its contribution to models of assertions. Given premises of the form
$\mathrm{A}$ is above $\mathrm{B}$.
$\mathrm{B}$ is above $\mathrm{C}$.

reasoners build a two- or three-dimensional mental model that satisfies the premises:

$$
\text { A }
$$$$
\text { B }
$$$$
\text { C }
$$

This model supports the conclusion $A$ is above $C$ and no model of the premises refutes this conclusion (see Johnson-Laird \& Byrne, 1991). Mental models, however, are not to be identified with visual images. Models are abstract, but they make it possible, in certain cases, to construct a visual image, from a particular point of view, of what a model represents (see, e.g., JohnsonLaird, 1998). On the other hand, a relation such as The hat is dirtier than the cup is easy to visualize, but it seems much less likely to be represented spatially. Subjectively, one seems to form an image of a dirty hat and an image of a less dirty cup. However, such an image contains a large amount of information that is irrelevant to the transitive inference. It puts an unnecessary load on working memory. In addition, reasoners have to isolate the information that is relevant to the inference, and in so doing, they might be sidetracked by irrelevant visual details. Hence, it is likely that a visual image of, say, a dirty hat and a dirty cup gets in the way of forming a representation that makes possible the transitive inference. This subjective impression motivates the following hypothesis.

THE VISUAL-IMAGERY-IMPEDANCE HYPOTHESIS. Relations that elicit visual images containing details that are irrelevant to an inference should impede the process of reasoning.

The phenomenal experience of the image, in other words, gets in the way of reasoning. Transitive inferences are so easy that it is unlikely that irrelevant visual detail should lead individuals into error, but it should slow down the process, since the inferential system has to find the pertinent information among the details. In contrast, if a relation yields a model that is pertinent to an inference, such as a spatial model in the case of spatial inference, it should speed up the process of reasoning, in comparison with relations that elicit neither images nor spatial representations. The aim of the following experiments was to compare this hypothesis with the orthodox view that visual imagery improves inference.

\section{EXPERIMENT 1}

Our first task was to carry out a preliminary study to determine whether the ease of visualizing a relation might be independent of the ease of forming a spatial representation of the relation. On the basis of our intuitions, we selected 15 pairs of relational terms (a relation and its converse) that might fall into the different categories. The relations included such pairs as cleaner-dirtier, uglier-prettier, heavier-lighter, and smarter-dumber, and the frequency of their usage was controlled using the norms in Francis and Kučera (1982). We formed 30 assertions on the basis of these relations, such as The cat is above the dog and The cat is smarter than the dog. All the assertions contained the same two nouns, cat and $d o g$. For half of the participants, cat was the subject of the sentence, and $d o g$ was the predicate; for the other half of the participants, the nouns were swapped around. Ten student volunteers at Princeton University, who were 
native English speakers, used two separate scales to rate the ease of forming visual images and the ease of forming spatial arrangements from each of the assertions. The two scales had seven points, ranging from very easy to very difficult. The assertions were presented in a different random order to each participant.

The ratings corroborated our intuitions. Relations do differ in the rated ease of forming both images and spatial representations of them, although we did not find any relations that were easy to envisage spatially that were not also easy to visualize. The ratings of assertions and their converses did not differ reliably, and we were able to select two pairs of each of three sorts from the set as a whole. These pairs and their ratings are presented in Table 1. The three sorts of relations were (1) relations such as above-below that were easy to envisage visually and spatially, which we henceforth refer to as visuospatial relations, (2) relations such as cleaner-dirtier that were easy to envisage visually but hard to envisage spatially, which we henceforth refer to as visual relations, and (3) relations such as better-worse that were hard to envisage both visually and spatially, which we henceforth refer to as control relations. The differences between the three sorts of pairs in Table 1 were statistically reliable (Friedman analysis of variance, $F=22.67$, $p<.001)$, whereas there were no significant differences between the pairs of relations within the three sorts.

In Experiment 1, we examined the three sorts of relational terms (visuospatial, visual, and control) in relational reasoning. If the visual-imagery-impedance hypothesis is correct, the visual relations should slow down the process of reasoning, in comparison with the visuospatial and control relations. But if the orthodox imagery hypothesis is correct, reasoning relies on images, when possible, and so the participants should perform better with visual relations.

\section{Method}

Participants. We tested 22 undergraduate students of Princeton University (mean age, 19.5 years; 12 female, 10 male), who received course credit for their participation.

Design and Materials. The participants acted as their own controls and evaluated 8 inferences based on each of the three sorts of relations (visuospatial, visual, and control). The resulting 24 infer-

Table 1

Norming Study 1: Mean Ratings for Ease of Forming a Visual Image and a Spatial Array of the Three Sorts of Relational Terms

\begin{tabular}{lcc}
\hline Relational Terms & $\begin{array}{c}\text { Visual } \\
\text { Ratings }\end{array}$ & $\begin{array}{c}\text { Spatial } \\
\text { Ratings }\end{array}$ \\
\hline Visual & & \\
$\quad$ cleaner-dirtier & 5.1 & 1.6 \\
$\quad$ fatter-thinner & 4.8 & 2.0 \\
Control & & \\
$\quad$ better-worse & 2.1 & 1.1 \\
$\quad$ smarter-dumber & 2.8 & 1.2 \\
$\quad$ Visuospatial & & \\
$\quad$ above-below & 5.3 & 5.4 \\
$\quad$ front-back & 5.2 & 5.3 \\
\hline
\end{tabular}

Note-The scales ranged from 1 (very difficult) to 7 (very easy). ences were presented in a different random order to each participant. Half of the inferences were three-term series, and half of them were four-term series. The relations in the inferences were those in Table 1. All inferences used the same nouns (dog, cat, ape, and for four-term inferences, bird) to minimize differences in imageability or processing times from one inference to another. Here is an example of a three-term inference with a valid conclusion:

The dog is cleaner than the cat.

The ape is dirtier than the cat.

Does it follow:

The dog is cleaner than the ape?

Here is an example of a four-term series inference with an invalid conclusion:

\section{The ape is smarter than the cat. \\ The cat is smarter than the dog. \\ The bird is dumber than the dog. \\ Does it follow: \\ The bird is smarter than the ape?}

Half the problems had valid conclusions, and half had invalid conclusions. In the first example above, cleaner and dirtier are used once in each premise, and cleaner occurs in the conclusion. But, in the experiment as a whole, the relational expressions (a relation and its converse; e.g., cleaner and dirtier) occurred equally often in each premise (first, second, and, where relevant, third) and in the conclusion.

Procedure. The participants were tested individually in a quiet room, using a laptop computer that administered the experiment. The presentation of the premises was self-paced and followed the separate-stage paradigm introduced by Potts and Scholz (1975). Each premise and putative conclusion was presented on a separate screen, and the participants proceeded from one to the next by pressing the space bar. The premises were presented in black letters, and the conclusion was presented in red letters. The participants were told to evaluate whether the conclusion followed necessarily from the premises. They made their responses by pressing either a "Yes" key or a "No" key on the keyboard. To familiarize the participants with the procedure prior to the experiment, there were four practice trials using other relations from the initial rating study. The program recorded the reading times for each of the premises separately and the response to the conclusion and its latency.

\section{Results and Discussion}

Table 2 presents, for all three of our experiments, the percentages of correct conclusions and their mean latencies for the different sorts of relational inferences. The present inferences were easy ( $89 \%$ correct overall), and there was no significant difference in the percentages of correct responses across the three sorts of relations (Friedman analysis of variance, $F=4.51, p>.1$ ). There was also no significant difference between accepting valid conclusions (91\% correct) and rejecting invalid conclusions ( $88 \%$ correct) and between the three-term and the four-term inferences. Hence, we pooled the results from these conditions. As Table 2 shows, the correct responses were faster for the visuospatial inferences than for the control inferences, which, in turn, were faster than those for the visual inferences. This predicted trend was significant (Page's $L=283, p<.05$; this test is nonparametric and appropriate for assessing the significance of a predicted trend in a within-subjects design; see Siegel \& Castellan, 1988). Although the following 
Table 2

Percentages of Correct Responses and Their Mean Response Latencies (RTs, in Seconds) in the Three Experiments as a Function of the Different Sorts of Relations: Visual Relations, Control Relations, Visuospatial Relations, and (in Experiment 3 Only) Spatial Relations

\begin{tabular}{|c|c|c|c|c|c|c|c|c|}
\hline \multirow[b]{2}{*}{ Experiment } & \multicolumn{2}{|c|}{$\begin{array}{c}\text { Visual } \\
\text { Relations }\end{array}$} & \multicolumn{2}{|c|}{$\begin{array}{c}\text { Control } \\
\text { Relations } \\
\end{array}$} & \multicolumn{2}{|c|}{$\begin{array}{c}\text { Visuospatial } \\
\text { Relations } \\
\end{array}$} & \multicolumn{2}{|c|}{$\begin{array}{c}\text { Spatial } \\
\text { Relations } \\
\end{array}$} \\
\hline & $\%$ & RT & $\%$ & RT & $\%$ & RT & $\%$ & RT \\
\hline 1 & 86 & 2.6 & 92 & 2.3 & 90 & 2.20 & - & - \\
\hline 2 & 77 & 3.37 & 67 & 2.64 & 68 & 2.46 & - & - \\
\hline 3 & 73 & 4.48 & 78 & 3.81 & 72 & 3.74 & 72 & 3.52 \\
\hline
\end{tabular}

comparisons are not orthogonal, the difference in latencies between the visuospatial and the control inferences was not significant, whereas the difference in latencies between the control and the visual inferences was significant (Wilcoxon test, $z=3.07, p<.002$ ).

The same trend also occurred in the times taken to read the premises (starting with the presentation of the first and ending with the disappearance of the last premise). Because all three premises yielded similar times, we pooled them together. The mean reading times were $6.6 \mathrm{sec}$ for the visual premises, $6.2 \mathrm{sec}$ for the control premises, and $6.0 \mathrm{sec}$ for the visuospatial tasks. The trend over the three relations was reliable (Page's $L=$ $284, p<.05)$.

The results corroborated the visual-imagery-impedance hypothesis and were contrary to the orthodox imagery hypothesis. In particular, the visual relations slowed reasoning, in comparison with control relations. But did the spatial relations speed up reasoning? The results are equivocal. On the one hand, the predicted trend was reliable; on the other hand, the difference between the visuospatial and the control relations was not reliable. One possible explanation is that the construction of a spatial model does not speed up reasoning but does not impede it either.

\section{EXPERIMENT 2}

The results of our first experiment might have depended on the use of transitive inferences in the reasoning problems. Because this sort of relational inference may favor certain strategies in reasoning, in our next experiment we examined the three sorts of relations in problems that called for a different sort of reasoning - namely, conditional reasoning. In the experiment, we again manipulated the three sorts of relations (visual, visuospatial, and control), but we also manipulated the difficulty of the inferences. Our aim was to avoid using inferences that were invariably easy and to examine the possibility of an interaction between difficulty and the three sorts of relations.

The participants had to evaluate conditional inferences in the form of modus ponens-for example,

If the ape is smarter than the cat, then the cat is smarter than the dog.

The ape is smarter than the cat.

\section{Does it follow:}

The ape is smarter than the dog?

In addition, however, the difficulty of the inference was manipulated by the use of converse relations. The easiest inferences were of the form exemplified in the preceding example:

Type 1: If $\mathrm{aRb}$ then $\mathrm{bRc}$

$\mathrm{aRb}$

$\mathrm{aRc}$ ?

The $a R b$ denotes a proposition asserting that a transitive relation, $R$, holds between two entities, $a$ and $b$. The difficulty of the inference was increased by using the converse relation, $R^{\prime}$, such as dumber, in the following two types of inference:

Type 2: If $a R b$ then $c^{\prime} b$

$\mathrm{aRb}$

aRc?

Type 3: If $b^{\prime}$ 'a then $b R c$

$b R^{\prime} a$

aRc?

And the hardest form of inference used two separate converse relations, one in the premise and one in the conclusion:

Type 4: If $b R^{\prime} a$ then $b R c$

$b R^{\prime} a$

$c R^{\prime} a$ ?

According to theories based on formal rules (e.g., Braine \& O'Brien, 1998; Rips, 1994), naive individuals are equipped with formal rules for modus ponens and transitive inferences. The mental model theory, however, postulates that reasoners make the easiest inference by constructing the following mental models for the conditional premise:

aRb bRc

where the two models are shown on separate rows. The second model denoted by the ellipsis has no explicit content but is just a placeholder for the possibilities in which the antecedent of the conditional is false. The second premise, $a R b$, in the easiest inference, eliminates this implicit model to leave only the first model. It yields the transitive conclusion $a R c$ (see, e.g., Johnson-Laird \& Byrne, 1991, for an account of how such transitive inferences are drawn). The use of converse relations is well known to increase the difficulty of transitive inferences (see, e.g., Clark, 1969; Huttenlocher, 1968). The visualimagery-impedance hypothesis predicts that the visual relations should be harder to deal with at all three levels of difficulty (Type $1<$ Types 2 and $3<$ Type 4 ).

\section{Method}

Participants. We tested a new sample of 20 students from the same population as those in Experiment 1. They received course credit for their participation. 
Design and Materials. The participants acted as their own controls and evaluated a valid and an invalid inference of the four types (1-4) for each of the three sorts of relations (visual, visuospatial, and control), making a total of 24 inferences. The inferences were presented in a different random order to each participant. Each inference concerned the same three noun phrases: the ape, the cat, and the dog. The procedure was identical to that of the first experiment, and there were four practice trials prior to the experiment proper.

\section{Results and Discussion}

Overall, the participants responded correctly to $82 \%$ of the valid problems and to $59 \%$ of the invalid problems. The effects of interest had the same patterns in both sorts of problem, and so we pooled the results. The trend concerning the effect of the converse relations on the percentages of correct responses felt short of significance: The easiest problems (Type 1) yielded $86 \%$ correct responses with a mean latency of $2.6 \mathrm{sec}$, the intermediate problems with one converse relation (Types 2 and 3 ) yielded $73 \%$ correct responses with a mean latency of $2.7 \mathrm{sec}$, and the hardest problems (Type 4) yielded $54 \%$ correct responses with a mean latency of $3.3 \mathrm{sec}$ (Page's $L=243, p>.05$, and $L=242, p>.05$, respectively). Likewise, the three sorts of relations did not have a significant effect on accuracy (see Table 2) at any level of difficulty, and there was no significant interaction between the two variables (Wilcoxon test, $z=0.58$, $p>.56)$.

Table 2 presents the mean latencies for the correct responses to the inferences based on the three sorts of relations. There was a reliable trend: Responses to the visuospatial inferences were faster than those to the control inferences, which, in turn, were faster than those to the visual inferences (Page's $L=255, p<.05$ ). The difference between the visuospatial inferences and the control inferences was not significant, but the control inferences were reliably faster than the visual inferences (Wilcoxon test, $z=2.46, p<.02$ ). There was no reliable interaction between the relations and the levels of difficulty in their effects on latencies (Wilcoxon test, $z=$ $0.75, p>.45$ ).

The reading times for the premises showed the same pattern: Visuospatial premises had a mean of $9.3 \mathrm{sec}$, the control premises had a mean of $10.3 \mathrm{sec}$, and the visual premises had a mean of $10.4 \mathrm{sec}$ (Page's $L=254, p<$ $.05)$. The participants were reliably faster to read the visuospatial premises than to read the control premises (Wilcoxon test, $z=2.05, p<.05$ ), but they were not reliably faster to read the control premises than the visual premises (Wilcoxon test, $z=0.18, p>.85$ ). The occurrence of converse relations in the premises had only a marginal effect on the reading times: The easiest problems (Type 1) had a mean of $9.7 \mathrm{sec}$, the middle-level problems (Types 2 and 3 ) had a mean of $10.5 \mathrm{sec}$, and the hardest problems (Type 4) had a mean of $9.8 \mathrm{sec}$ (Friedman analysis of variance, $F=5.70, p>.058$ ). Likewise, there was no significant interaction between the two variables (Friedman analysis of variance, $F=$ $1.20, p>.54)$.
As was expected, converse relations showed a tendency, not quite reliable, to impair both accuracy and speed of inference. The principal result, however, was the confirmation that the nature of the relations influenced reasoning time. Inferences based on visual relations again took more time, in comparison with control relations that were difficult to visualize. Once again, the visuospatial relations did not speed up the process of inference, or at least the time to respond to the presentation of the last premise in a problem, but reasoners did read the visuospatial premises faster. This result suggests that visuospatial relations can facilitate the process of understanding the premises - that is, constructing a mental representation of them.

\section{EXPERIMENT 3}

What happens if a relation is easy to envisage spatially but not easy to visualize? Our previous rating study failed to discover such relations. If, as our findings suggest, the imageability of the materials leads to an impairment of reasoning performance, whereas the possibility of spatially envisaging the materials speeds up comprehension or reasoning, tasks based on purely spatial relations - that is, those that are not so easy to visualize - should be processed most quickly. We therefore renewed our search for such relations in order to study inferential performance with them.

To find purely spatial relations, 12 native German speakers, who were students at the University of Freiburg, completed a questionnaire that was similar to the one in our first rating study. Again, the participants rated the ease of forming visual images and spatial representations for a set of relational assertions. We included the relations from the first study but also added some relations that seemed easy to envisage spatially but difficult to visualize: earlier-later, older-younger, hotter-colder, stronger-weaker,faster-slower, ancestor-of-descendantof, and further-north-further-south (which are single words in German). The results replicated those from the study at Princeton University and yielded the same visuospatial relations, visual relations, and control relations. However, no purely spatial relations emerged from the study. We concluded that the use of separate ratings might not be sensitive enough to reveal them. We therefore carried out a study using a different rating procedure.

The participants rated on a single bipolar 7-point scale whether each relation evoked a visual or, else, a spatial representation. The poles of the scale were labeled visual and spatial, respectively. The instructions stated that a visual representation is a vivid mental image that may include concrete people, objects, colors, or shapes and that it can be similar to a real perception, whereas a spatial representation is a more abstract layout that represents something on a scale, an axis, or a spatial array. Although the points on the scale were not numbered for the participants, we coded the spatial pole as -3 and the visual pole as +3 . We used a set of 35 relations, which were controlled for frequency of usage in German (using the Projekt Deutscher Wortschatz, although the spatial rela- 
Table 3

Norming Study 2: Mean Ratings for Ease of Forming a Visual Image and a Spatial Array of the Four Sorts of Relational Terms

\begin{tabular}{lc}
\hline Relational Term & Rating \\
\hline Visual & \\
cleaner-dirtier & 2.1 \\
fatter-thinner & 1.6 \\
Control & \\
better-worse & -0.7 \\
smarter-dumber & -0.7 \\
Visuospatial & \\
above-below & -0.9 \\
front-back & -0.8 \\
Spatial & \\
north-south & -2.7 \\
ancestor-descendant & -0.9 \\
\hline
\end{tabular}

Note-The mean ratings were on a single scale that ranged from easy to form a visual image $(+3)$ through zero to easy to form a spatial representation $(-3)$

tions had a slightly lower frequency of usage). Twenty students participated in the rating study.

Table 3 presents the main results of the ratings. The results identified two pairs of spatial relations: ancestorof-descendant-of and further-north-further-south (in German, nördlicher and südlicher, which do not require the comparative further). The ratings of the four classes of words differed significantly (Friedman analysis of variance, $F=38.33, p<.001$ ). The spatial relations differed reliably from the control relations (Wilcoxon test, $z=3.16, p<.002)$. However, there was no difference between the ratings of the visuospatial and the control relations on the scale; we distinguish them solely on the basis of their ratings on separate scales.

In the third experiment, we examined relational reasoning with the four sorts of relations. The visual-imageryimpedance hypothesis predicts that the visual relations should slow down reasoning with the visual relations. If the construction of a spatial model speeds up reasoning, even in the absence of visualization, both the spatial and the visuospatial relations should speed up reasoning. In sum, the four relations should show the following trend in increasing latencies for reading and reasoning: spatial, visuospatial, control, and visual.

\section{Method}

Participants. Twenty-four undergraduate students of Freiburg University (mean age, 22.4 years; 14 female, 10 male) participated in the experiment. They either were paid or received course credit for their participation.

Design and Materials. The materials consisted of 16 three-term and 16 four-term series inferences that were structured identically to the materials in the first experiment. There were four sorts of problems (spatial, visuospatial, visual, and control). The spatial relations were ancestor-of-descendant-of and further-north-further-south (single words in German). There were two valid and two invalid inferences of each of the four sorts in both the three-term and the fourterm series inferences (the total of 32 inferences). The participants acted as their own controls and evaluated inferences of all the varieties presented in a different random order to each participant.
Procedure. The participants were tested individually in a quiet room. They sat at a $\mathrm{PC}$ with a 14-in. monitor, and the premises were presented one at a time on a sequence of screens (in black letters) followed by a putative conclusion (in red letters). The participants were told to evaluate whether the conclusion followed logically from the premises. They made their responses by pressing the appropriate key on the keyboard, and the computer recorded the response and latency. The procedure was identical to that of the other experiments, and prior to the experiment, there were four practice trials using relations other than those in the experiment proper.

\section{Results and Discussion}

Overall, the participants responded correctly to $72 \%$ of the valid inferences and $76 \%$ of the invalid inferences. There was no significant difference in the error rates for the four sorts of relations (Friedman analysis of variance, $F=2.63, p>.45$ ). Table 2 presents the mean latencies for the correct responses to the four sorts of relational inferences. These latencies corroborated the predicted trend: spatial relations $<$ visuospatial relations $<$ control relations < visual relations (Page's $L=648, z=3.40, p<$ $.05)$. As Table 2 suggests, however, the principal effect is that visual relations slow down reasoning, relative to the other three relations (Wilcoxon test, $z=2.46, p<.015$ ).

As in the first experiment, the mean reading time for the visual premises $(6.1 \mathrm{sec})$ was longer than that for the visuospatial premises $(5.6 \mathrm{sec})$ and the control premises $(5.4 \mathrm{sec})$. However, the longest reading times for the premises were measured for the spatial relations $(6.3 \mathrm{sec})$, but north-south relations were read relatively slowly $(6.8$ $\mathrm{sec})$, whereas ancestor-descendant relations were read relatively quickly $(5.8 \mathrm{sec})$. For heuristic purposes, we made the following statistical comparisons even though they are not orthogonal: Visual relations impeded reading times in comparison with control relations (Wilcoxon test, $z=2.49, p<.01)$; spatial relations impeded reading times in comparison with control relations (Wilcoxon test, $z=3.97, p<.001$ ); visuospatial relations did not differ reliably from control relations (Wilcoxon test, $z=$ $1.06, p>.29$ ).

In summary, visual relations impeded reasoning in comparison with the other three sorts of relations. There was a trend that favored the spatial relations, but they did not differ reliably from the control relations. Similarly, the reading times for premises showed that the visual relations took longest to read. Unlike our previous experiment, however, the visuospatial relations showed no advantage in reading time over the control relations, and our newfound spatial relations had longer reading times. It may take reasoners longer to envisage representations of relative compass points and kinship relations, but these terms are less frequently used than the other relations. In fact, the majority of the participants reported that the spatial relations evoked "mental pictures"-for instance, a topographic map for the north-south relations.

\section{GENERAL DISCUSSION}

Our starting point was the conjecture that the conflicting results in the literature on images and deductive 
reasoning arose from a failure to distinguish between visual and spatial modes of representation. A preliminary rating study enabled us to identify visuospatial relations, such as above-below, which are easy to envisage both visually and spatially, visual relations, such as cleanerdirtier, which are easy to envisage visually but hard to envisage spatially, and control relations, such as betterworse, which are hard to envisage both visually and spatially. In a subsequent rating study, we were able to identify some spatial relations, such as further-north-further-south, that are harder to envisage visually than to envisage spatially. All three of our experiments showed that visual relations significantly impeded the process of reasoning by slowing it down. The results also showed a trend in which visuospatial and spatial relations sped up reasoning, in comparison with control relations. However, the direct comparison of this difference was not significant in any of our experiments.

The impeding effect of visual relations appears to resolve at least some of the apparently inconsistent results in the literature. The group of studies that found a facilitating effect of "imagery" tended to use materials that differed in the ease of constructing spatial representations, whereas the group of studies that found no such effect, or an impeding effect of imageability, tended to use materials that evoked visual representations. In the first group (facilitating effect of "imagery"), the study by De Soto et al. (1965) examined the extent to which different relations evoked a representation with a spatial axis. Spatial relations, such as above and below, elicited such representations, as did such control relations as better and worse, whereas visual relations, such as lighter and darker, elicited no directional preference. The spatial and control relations facilitated reasoning, in comparison with the visual relations. Shaver et al. (1975) investigated transitive inferences with the three sorts of relations above, has lighter hair than, and better than. They reported that participants made more errors in the hair color problems (visual) than in the better-than problems (an instance of our control relations), which in turn were harder than the problems with above (visuospatial).

In the second group of studies (no imageability effect or an impeding effect), the study by Richardson (1987) varied the imageability of faces, a visual variable rather than a spatial one. The manipulation had no effect on reasoning. Similarly, Johnson-Laird et al. (1989) examined reasoning with three transitive relations: equal in height, in the same place as, and related to (in the sense of kinship). The relations had no effect on reasoning accuracy. In our analysis, the three relations can all be represented spatially. The same explanation can account for Clement and Falmagne's (1986) results. They studied conditional reasoning and varied the imageability and availability of pertinent knowledge. These two factors interacted, but in the direct comparison there was no difference between problems based on statements such as If a man walks his golden retriever, then he gets upset about his insect bite, which were assumed to be highly visual, and problems based on statements such as If the man takes an economic perspective, then he uses the new memory technique, which were assumed to be difficult to visualize. The study has been criticized on methodological grounds (Evans, 1980). And in hindsight, it appears to have confounded imageability with spatial and other factors. Finally, Egan and Grimes-Farrow (1982) gathered retrospective reports from participants who had drawn three-term series inferences. Those who reported using visual imagery performed worse than those who reported using other, more abstract strategies.

According to Paivio (1975), the decisive criterion for a particular sort of mental representation is the disruption of performance by a concurrent task relying on the same sort of representation. Klauer, Stegmaier, and Meiser (1997) found that a task in which the participants had to remember a spatially organized sequence of keys impaired conditional reasoning. Likewise, Knauff, Jola, and Strube (2001; cf. also Knauff, Jola, Strube, Rauh, \& Schlieder, 2002) examined the disruption of three-term series inferences by secondary tasks that were either visual or auditory and either spatial or nonspatial. Only the spatial tasks disrupted inference, regardless of whether they were auditory or visual.

Studies using brain imaging methods such as positron emission tomography and functional magnetic resonance imaging also count against the use of visual images in reasoning. In a study by Knauff, Mulack, Kassubek, Salih, and Greenlee (2002), both conditional and relational reasoning activated a bilateral parietal-frontal network distributed over parts of the prefrontal cortex, the inferior and superior parietal cortex, and the precuneus, whereas no significant activation was measured in the occipital cortex, usually activated by visual imagery (Kosslyn et al., 1993; Kosslyn et al., 1999; Kosslyn, Thompson, \& Alpert, 1997; Sabbah et al., 1995; a contrasting result is reported in Knauff, Kassubek, Mulack, \& Greenlee, 2000). In fact, reasoning activated regions of the brain that make up the "where-pathway" of spatial perception and working memory (e.g., Smith et al., 1995; Ungerleider \& Mishkin, 1982). In contrast, the "what-pathway" that processes visual features such as shape, texture, and color (cf. also Landau \& Jackendoff, 1993; Rueckl et al., 1989; Ungerleider, 1995) seems not to be involved. Other studies have corroborated these findings. Prabhakaran, Smith, Desmond, Glover, and Gabrieli (1997) studied Raven's progressive matrices and found (for inductive reasoning) increased activity in right frontal and bilateral parietal regions. Osherson et al. (1998) compared inductive and deductive reasoning and found that the latter increased activation in right-hemisphere parietal regions. Goel and Dolan (2001) studied concrete and abstract three-term relational reasoning and found activation in a parietaloccipital-frontalnetwork. And Kroger, Cohen, and JohnsonLaird (2002) found that reasoning, in contrast to mental arithmetic based on the same assertions, activated right frontal areas often associated with spatial representation.

What might cause visual imagery to impede reasoning? Theories of reasoning based on formal rules postulate that rules of inference operate on sentences or their 
logical forms (Braine \& O’Brien, 1998; Rips, 1994). This syntactic approach, however, does not account for the effects of content of inference and does not immediately suggest an explanation of impedance. Theories based on visual images postulate that they are akin to percepts (Berendt, 1996; Finke, 1989; Kosslyn, 1980), and thus memory of an image can be confused with memory for a percept (Johnson \& Raye, 1981). However, a theory that relied on visual imagery as the medium for reasoning would be implausible, because individuals can reason about relations that they cannot visualize. Similarly, such a theory cannot readily explain why relations that are easy to envisage visually impeded reasoning.

In contrast to visual images, mental models can represent any possible situation and can abstract away from such visual details as colors, textures, and shapes (JohnsonLaird, 1983; Johnson-Laird \& Byrne, 1991; Knauff, 1999; Knauff, Rauh, Schlieder, \& Strube, 1998). They can represent spatial relations. For example, take the premises

The cat is above the ape.

The dog is below the ape.

These premises elicit a model representing the relative positions of the three individuals:
cat
ape
$\operatorname{dog}$

The model supports the conclusion

The cat is above the dog.

There is no counterexample to this conclusion-that is, no model that satisfies the premises but refutes the conclusion. Hence, the conclusion is valid. Spatial inferences that call for multiple models are, as predicted, more difficult than those based on a single model (Byrne \& Johnson-Laird, 1989). Mental models, however, can also represent class inclusion, temporal order, and abstract relations, such as ownership (see, e.g., JohnsonLaird \& Byrne, 1991). The effects of content can facilitate inferences in certain cases and impede them in other cases (Johnson-Laird \& Byrne, in press). Likewise, a visual relation, such as dirtier than, can elicit a vivid visual detail, such as an animal caked with mud, that is irrelevant to an inference. It will then take additional time to retrieve the information needed to construct the appropriate mental model for making the inference.

Is there an alternative explanation for our results? If, for example, the visual relations had differed in frequency of usage from the other relations, would that factor explain the visual impedance of inference? Certainly, rarely used words might have slowed down the reading times for the premises, but it seems unlikely that once the premises had been interpreted, this factor would have impeded reasoning itself. A different possibility is that relations differ in the degree to which they imply transitivity. Spatial rela- tions are unequivocal, but visual relations might be more dubious. Given, say, the following premises:

The cat is fatter than the ape.

The ape is fatter than the dog.

reasoners might have wondered whether the fatness of cats, apes, and dogs is commensurable. The claim that, say, an elephant is thin is relative to elephants, and so it is sensible to assert that a thin elephant is fatter than a fat dog. The criterion for fatness shifts from one animal to another. This factor might have confused the reasoners in our experiment and impeded their inferences with the visual relations. Contrary to this account, however, the participants drew just as many transitive inferences from the visual relations as they did from the other relations. Yet another possible factor is the degree to which the premises accord with the participants' existing beliefs. For example, the preceding premise (the cat is fatter than the ape) might strike some individuals as implausible. In the experiments as a whole, each such implausible premise is matched with one using the converse relation (the cat is thinner the ape), and so this factor seems unlikely to account for our results.

In conclusion, our results suggest that the content of assertions can affect the process of inference. If the content yields information relevant to an inference, as it does with visuospatial relations, comprehension and reasoning proceed smoothly and may even be slightly faster than with other sorts of content. But if the content yields visual images that are irrelevant to an inference, as it does with visual relations, reasoning is impeded and reliably takes longer. The vivid details in the mental image interfere with thinking, much as they did for the character from William Golding's novel in the epigraph to this article.

\section{REFERENCES}

Berendt, B. (1996). Explaining preferred mental models in Allen inferences with a metrical model of imagery. In G. N. Cottrell (Ed.) Proceedings of the Eighteenth Annual Conference of the Cognitive Science Society (pp. 489-494). Mahwah, NJ: Erlbaum.

Braine, M. D. S., \& O'Brien, D. P. (Eds.) (1998). Mental logic. Mahwah, NJ: Erlbaum.

Byrne, R. M. J., \& Johnson-Laird, P. N. (1989). Spatial reasoning. Journal of Memory \& Language, 28, 564-575.

Clark, H. H. (1969). Linguistic processes in deductive reasoning. Psychological Review, 76, 387-404.

Clement, C. A., \& Falmagne, R. J. (1986). Logical reasoning, world knowledge, and mental imagery: Interconnections in cognitive processes. Memory \& Cognition, 14, 299-307.

De Soto, L. B., London, M., \& Handel, M. S. (1965). Social reasoning and spatial paralogic. Journal of Personality \& Social Psychology, 2, 513-521.

D'Esposito, M., Aguirre, G. K., Zarahn, E., Ballard, D., Shin, R. K., \& LEASE, J. (1998). Functional MRI studies of spatial and nonspatial working memory. Cognitive Brain Research, 7, 1-13.

Egan, D. E., \& Grimes-Farrow, D. D. (1982). Differences in mental representations spontaneously adopted for reasoning. Memory \& Cognition, 10, 297-307.

Evans, J. S. B. T. (1980). Thinking: Experimental and information processing approaches. In G. Claxton (Ed.), Cognitive psychology: New directions (pp. 275-299). London: Routledge. 
Finke, R. A. (1989). Principles of mental imagery. Cambridge, MA: MIT Press.

Francis, W. N., \& KuČERA, H. (1982). Frequency analysis of English usage: Lexicon and grammar. Boston: Houghton Mifflin.

Goel, V., \& Dolan, R. J. (2001). Functional neuroanatomy of threeterm-relational reasoning. Neuropsychologia, 39, 901-909.

Golding, W. (1955). The inheritors. New York: Harcourt Brace.

Huttenlocher, J. (1968). Constructing spatial images: A strategy in reasoning. Psychological Review, 75, 550-560.

Johnson, M. K., \& RAYE, C. L. (1981). Reality monitoring. Psychological Review, 88, 67- 85 .

Johnson-Laird, P. N. (1983). Mental models. Cambridge: Cambridge University Press

JoHNSON-LAIRD, P. N. (1998). Imagery, visualization, and thinking. In J. Hochberg (Ed.), Perception and cognition at century's end (pp. 441467). San Diego: Academic Press.

Johnson-Laird, P. N., \& Byrne, R. M. J. (1991). Deduction. Hove, U.K.: Erlbaum.

Johnson-Laird, P. N., \& Byrne, R. M. J. (in press). Conditionals: A theory of meaning, pragmatics, and inference. Psychological Review.

Johnson-Laird, P. N., BYrne, R. M. J, \& Tabossi, P. (1989). Reasoning by model: The case of multiple quantifiers. Psychological Review, 96, 658-673.

Klauer, K. C., Stegmaier, R., \& Meiser, T. (1997). Working memory involvement in propositional and spatial reasoning. Thinking \& Reasoning, 3, 9-47.

KnaufF, M. (1999). The cognitive adequacy of Allen's interval calculus for qualitative spatial representation and reasoning. Spatial Cognition \& Computation, 1, 261-290.

Knauff, M., Jola, C., \& Strube, G. (2001). Spatial reasoning: No need for visual information. In D. Montello (Ed.), Spatial information theory: Proceedings of COSIT'01 (pp. 447-457). New York: Springer-Verlag.

Knauff, M., Jola, C., Strube, G., Rauh, R, \& Schlieder, C. (2002). Spatial representations can be the basis of reasoning. Manuscript submitted for publication.

Knauff, M., Kassubek, J., Mulack, T., \& Greenlee, M. W. (2000) Cortical activation evoked by visual mental imagery as measured by functional MRI. NeuroReport, 11, 3957-3962.

Knauff, M., Mulack, T., KassubeK, J., Salih, H. R. \& Greenlee, M. W. (2002). Spatial imagery in deductive reasoning: A functional MRI. Cognitive Brain Research, 13, 203-212.

Knauff, M., Rauh, R., Schlieder, C., \& Strube, G. (1998). Mental models in spatial reasoning. In C. Freksa, C. Habel, \& K. F. Wender (Eds.), Spatial cognition: An interdisciplinary approach to representing and processing spatial knowledge (Lecture Notes in Computer Science, Bd. 1404, pp. 267-291). Berlin: Springer-Verlag.

KossLyn, S. M. (1980). Image and mind. Cambridge, MA: Harvard University Press.

Kosslyn, S. M. (1994). Image and brain. Cambridge, MA: MIT Press.

Kosslyn, S. M., Alpert, N. M., Thompson, W. L., Maljkovic, V., Weise, S. B., Chabris, C. F., Hamilton, S. E., Rauch, S. L., \& Buonanno, F. S. (1993). Visual mental imagery activates topographically organized visual cortex: PET investigations. Journal of Cognitive Neuroscience, 5, 263-287.

Kosslyn, S. M., Pascual-Leone, A., Felician, O., Camposano, S., Keenan, J. P., Thompson, W. L., Ganis, G., Sukel, K. E., \& Alpert, N. M. (1999). The role of area 17 in visual imagery: Convergent evidence from PET and rTMS. Science, 2, 167-170.

Kosslyn, S. M., Thompson, W. L., \& Alpert, N. M. (1997). Neural systems shared by visual imagery and visual perception: A positron emission tomography study. NeuroImage, 6, 320-334.

Kroger, J., Cohen, J., \& Johnson-Laird, P. N. (2002). A double dis- sociation between logic and mathematics: A functional magnetic resonance imaging study. Manuscript submitted for publication.

LANDAU, B., \& JACKENDOFF, R. (1993). "What" and "where" in spatial language and spatial cognition. Behavioral \& Brain Sciences, 16, 217-265

LogIe, R. H. (1995). Visuo-spatial working memory. Hove, U.K.: Erlbaum. Newcombe, F., \& RAtclifF, G. (1989). Disorders of visuospatial analysis. In F. Boller \& J. Grafman (Eds.), Handbook of neuropsychology (pp. 333-356). Amsterdam: Elsevier.

Newcombe, F., Ratcliff, G., \& Damasio, H. (1987). Dissociable visual and spatial impairments following right posterior cerebral lesions: Clinical, neuropsychological and anatomical evidence. $\mathrm{Neu}$ ropsychologia, 18, 149-161.

Newstead, S. E., Pollard P., \& Griggs, R. A. (1986). Response bias in relational reasoning. Bulletin of the Psychonomic Society, 24, 95-98.

Osherson, D., Perani, D., Cappa, S., Schnur, T., Grassi, F., \& FAZIO, F. (1998). Distinct brain loci in deductive versus probabilistic reasoning. Neuropsychologia, 36, 369-376.

Paivio, A. (1975). Perceptual comparisons through the mind's eye. Memory \& Cognition, 3, 635-647.

Pearson, D. G., Logie, R. H., \& Gilhooly, K. J. (1999). Verbal representations and spatial manipulation during mental synthesis. European Journal of Experimental Psychology, 11, 295-314.

PotTs, G. R. \& Scholz, K. W. (1975). The internal representation of a three-term series problem. Journal of Verbal Learning \& Verbal Behavior, 14, 439-452.

Prabhakaran, V., Smith, J. A., Desmond, J. E., Glover, G. H., \& GABRIELI, J. D. (1997). Neural substrates of fluid reasoning: An fMRI study of neocortical activation during performance of the Raven's Progressive Matrices Test. Cognitive Psychology, 33, 43-63.

RichaRdSON, J. T. E. (1987). The role of mental imagery in models of transitive inference. British Journal of Psychology, 78, 189-203.

RIPS, L. J. (1994). The psychology of proof. Cambridge, MA: MIT Press.

Rueckl, J. G., CAve, K. R. \& Kosslyn, S. M. (1989). Why are "what" and "where" processed by separate cortical visual systems? A computational investigation. Journal of Cognitive Neuroscience, 1, 171-186.

Sabbah, P., Simond, G., Levrier, O., Habib, M., Trabaud, V., Murayama, N., Mazoyer, B. M., Briant, J. F., Raybaud, C., \& Salamon, G. (1995). Functional magnetic resonance imaging at $1.5 \mathrm{~T}$ during sensorimotor and cognitive tasks. European Journal of Neurology, 35, 131-136.

Shaver, P., Pierson, L., \& LANG, S. (1975). Converging evidence for the functional significance of imagery in problem solving. Cognition, $\mathbf{3}$, 359-375.

Shepard, R. N., \& Cooper, L. A. (1982). Mental images and their transformations. Cambridge, MA: MIT Press.

Siegel, S., \& CASTEllan, N. J., JR. (1988). Nonparametric statistics for the behavioral sciences (2nd ed.). New York: McGraw-Hill.

Smith, E. E., Jonides, J., Koeppe, R. A., Awh, E., Schuhmacher, E. H. \& Minoshima, S. (1995). Spatial versus object working memory: PET investigations. Journal of Cognitive Neuroscience, 7, 337-356.

STERnberG, R. J. (1980). Representation and process in linear syllogistic reasoning. Journal of Experimental Psychology: General, 109, 119-159.

UNGERLEIDER, L. G. (1995). Functional brain imaging studies of cortical mechanisms for memory. Science, 270, 769-775.

Ungerleider,L. G., \& MishKIN, M. (1982). Two cortical visual systems. In D. J. Ingle, M. A. Goodale, \& R. J. W. Mansfield (Eds.), Analysis of visual behaviour (pp. 549-586). Cambridge, MA: MIT Press.

(Manuscript received May 15, 2001; revision accepted for publication December 3, 2001.) 